\title{
Effects of sleep bruxism on functional and occlusal parameters: a prospective controlled investigation
}

\author{
Michelle Alicia Ommerborn ${ }^{1}$, Maria Giraki ${ }^{1}$, Christine Schneider ${ }^{2}$, Lars Michael Fuck ${ }^{3}$, Jörg Handschel ${ }^{4}$, \\ Matthias Franz ${ }^{2}$, Wolfgang Hans-Michael Raab ${ }^{1}$ and Ralf Schäfer ${ }^{2}$
}

This study was conducted to verify the results of a preceding retrospective pilot study by means of a prospective controlled investigation including a larger sample size. Therefore, the aim of this clinical investigation was to analyze the relationship between sleep bruxism and several functional and occlusal parameters. The null hypothesis of this study was that there would be no differences among sleep bruxism subjects and non-sleep bruxism controls regarding several functional and occlusal parameters. Fifty-eight sleep bruxism subjects and 31 controls participated in this study. The diagnosis sleep bruxism was based on clinical criteria of the American Academy of Sleep Medicine. Sixteen functional and occlusal parameters were recorded clinically or from dental study casts. Similar to the recently published retrospective pilot study, with a mean slide of $0.77 \mathrm{~mm}$ (s.d., $0.69 \mathrm{~mm}$ ) in the sleep bruxism group and a mean slide of $0.4 \mathrm{~mm}$ (s.d., $0.57 \mathrm{~mm}$ ) in the control group, the evaluation of the mean comparison between the two groups demonstrated a larger slide from centric occlusion to maximum intercuspation in sleep bruxism subjects (Mann-Whitney $U$-test; $P=0.008$ ). However, following Bonferroni adjustment, none of the 16 occlusal and functional variables differed significantly between the sleep bruxism subjects and the non-sleep bruxism controls. The present study shows that the occlusal and functional parameters evaluated do not differ between sleep bruxism subjects and non-sleep bruxism subjects. However, as the literature reveals a possible association between bruxism and certain subgroups of temporomandibular disorders, it appears advisable to incorporate the individual adaptive capacity of the stomatognathic system into future investigations.

International Journal of Oral Science (2012) 4, 141-145; doi:10.1038/ijos.2012.48; published online 31 August 2012

Keywords: dental occlusion; functional parameters; prospective study; sleep bruxism

\section{INTRODUCTION}

Sleep bruxism has characteristically been defined by the American Academy of Sleep Medicine as 'an oral activity characterized by grinding or clenching of the teeth during sleep, usually associated with sleep arousals'. ${ }^{1}$ Although numerous investigations stress the assumption of a central causation, such as neuropathophysiology and psychology, ${ }^{2-6}$ a detailed clarification regarding the aetiology of sleep bruxism is still lacking. To date, most authors correspond with the supposition of a multifactorial development of sleep bruxism. ${ }^{7-9}$ During a few years, explanatory models in the field of the dental profession, such as occlusal interferences or variances in the orofacial anatomy, ${ }^{10-12}$ are thought to be of inferior, possibly without any, relevance in the development of sleep bruxism activity. ${ }^{13}$ For this reason, the perception in respect of the relationship between sleep bruxism and the stomatognathic system has changed. ${ }^{13-14}$ Consequently, sleep bruxism is supposed to be induced centrally, whereas the effects of this parafunctional activity are predominantly found in the stomatognathic system. Undoubtedly, apart from associated effects, such as unpleasant muscle and tooth sensations, limitation of jaw movements, oral and facial pain, and headache; ${ }^{1}$ tooth attrition, fractured cusps or entire teeth, shiny spots on restorations are well-known to be the most frequently occurring effects, in particular, on the dental hard tissue. ${ }^{15}$ Moreover, a possible association between bruxism and temporomandibular disorders (TMDs) is supposed, but the available literature reveals heterogeneous data. ${ }^{9,16-18}$ For some past time, by indicating a relationship between bruxism and posture, diverse studies draw the attention to a more extensive context. ${ }^{19-25}$ However, the authors of a recently published review come to the conclusion that a scientific prove to support a cause-effect relationship is still missing. ${ }^{26}$

To gain information on the clinical interaction among sleep bruxism and several occlusal and functional parameters by using a more clinically feasible, but investigator-independent assessment tool for sleep bruxism diagnosis, in a recently published pilot study 16 occlusal and functional parameters were recorded and compared with those obtained from a non-sleep bruxism control group. ${ }^{27}$ By means of a retrospective study design, the diagnosis of sleep bruxism was performed using a specifically conceived computer-based analyzing method for the Bruxcore Bruxism-Monitoring Device that has previously evaluated regarding its ability to objectively differentiate between sleep bruxism subjects and an adequate non-sleep bruxism

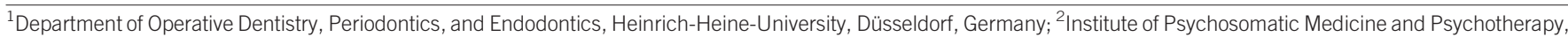

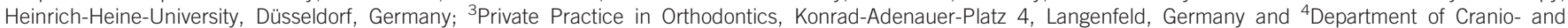
Maxillofacial Surgery, Heinrich-Heine-University, Düsseldorf, Germany

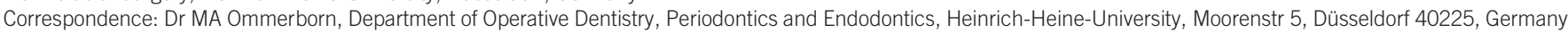
E-mail: Ommerborn@med.uni-duesseldorf.de

Received 17 April 2012; accepted 30 May 2012 
control group. ${ }^{28}$ Considering all variables tested, with an approximately $0.5 \mathrm{~mm}$ larger slide from centric occlusion (CO) to maximum intercuspation (MI) in the sleep bruxism group, the results solely demonstrated a statistically significant group difference regarding the length of the slide from CO to MI.

In order to verify the results of the former pilot study, as a next step a prospective controlled investigation including a larger sample size has been conducted using the clinical criteria of the American Academy of Sleep Medicine for sleep bruxism diagnosis. ${ }^{1,6}$ Therefore, the aim of the present prospective study was to analyze the relationship between sleep bruxism and several functional and occlusal parameters. The null hypothesis of this study was that there would be no differences among a sample of sleep bruxism subjects and a non-sleep bruxism control group regarding several functional and occlusal parameters.

\section{MATERIALS AND METHODS}

\section{Selection of subjects}

The entire sample consisted of 91 subjects, of whom 58 were females and 33 were men with a mean age of 28.37 years (standard deviation (s.d.), 4.89 years; age range, 20-39 years). They were all German native speakers and responded to announcements in local newspapers and placards on campus. Each participant was screened following a thorough dental examination. As applied in former investigations, ${ }^{5-6,28-30}$ the diagnosis of sleep bruxism was based on the clinical criteria of the American Academy of Sleep Medicine. ${ }^{1}$ Individuals who met the following criteria were included in the sleep bruxism group: healthy adults, aged between 20 and 40 years, sleeping partner reports of grinding sounds during the night in the last 6 months, and at least one of the following symptoms: self-report of muscle fatigue or tenderness on awakening, the presence of tooth wear to at least the magnitude of dentin exposure, ${ }^{31}$ and masseter hypertrophy upon voluntary forceful clenching. ${ }^{1,17}$

Exclusion criteria were: current dental treatment, severe psychological disorder and/or the use of antipsychotic psychotropic drugs, central nervous system and/or peripheral nervous system disorders, more than two missing molars (excluding third molars), the presence of prosthesis or extensive prosthetic restorations and the presence of gross malocclusion. Healthy adults, from whom sleep bruxism could be excluded, represented the control group. Exclusion criteria were the same as for the sleep bruxism group as well as any signs and symptoms of sleep bruxism. All subjects gave informed consent to the procedures approved by the Institutional Human Subjects Ethics Committee (Heinrich-Heine-University of Duesseldorf).

\section{Functional and occlusal parameters}

At first, each of the 91 participants had a thorough dental examination which was performed by one trained dentist of the department. Similar to the recently published pilot study which used the newly developed computer-based analyzing method for calculating abrasion on the Bruxcore Bruxism-Monitoring Device as measure for sleep bruxism activity, ${ }^{27-28}$ the following functional and occlusal parameters were clinically recorded by means of a digital calliper: vertical (overbite) and horizontal (overjet) overlap of the maxillary and mandibular right central incisors, maximum active mouth opening, maximum active right and left lateral movement of the mandible, maximum protrusive movement of the mandible, the presence of a slide from $\mathrm{CO}$ to $\mathrm{MI}^{32}$ and, if present, the length of the slide from $\mathrm{CO}$ to MI. The recording of the slide has been performed as described previously. ${ }^{27,32-34}$ Moreover, the resiliency of the left and right TMJ, ${ }^{35}$ as well as the presence of lesions related to lip and/or cheek biting were determined. Furthermore, the Angle's Classification of malocclusion, recorded on the right and the left side for the canines and for the first molars, ${ }^{36}$ as well as the anterior crowding in the mandible (classified on a five-point scale: 0 , no crowding; $1,1-3 \mathrm{~mm}$ of crowding; 2, 3-5 mm; 3, 5-7 $\mathrm{mm} ; 4,>7 \mathrm{~mm}$ ) were identified from dental study casts. ${ }^{13,27}$ Due to either missing canines or first molars in some patients, the sample size varied between 68 and 65 participants.

\section{Statistical analysis}

Statistical analyses were performed using the statistical software SPSS version 19.0 (IBM Corp., Armonk, NY, USA). Normal distribution of the variables was verified by means of the Kolmogorov-Smirnov test combined with the assessment of histograms. The Pearson Chi-square test was applied to determine the significance of differences between two independent groups when data consisted of frequencies in qualitative variables. If a normal distribution of the data was found, the independent samples Student's $t$-test was applied for the analysis of mean differences between both groups in quantitative variables. For all quantitative variables with lacking normal distribution, differences were evaluated by means of the nonparametric Mann-Whitney $U$-test. When using the Mann-Whitney $U$-test, the adequate statistical values are the mean ranks and the sum of ranks. However, to improve the comparability of the obtained results, data are presented as means and s.d.. For all statistical analyses, an $\alpha$-error probability level of $P<0.05$ was defined as the statistical significant level. To correct the observed significance level according to the number of comparisons made, Bonferroni adjustment was applied. Since 19 comparisons were performed including sociodemographic data, the Bonferroni-adjusted probability level amounted to $P<0.003$.

\section{RESULTS}

Comparisons of the two groups showed no significant differences regarding age, gender and education (Table 1). Furthermore, in Tables 2 and 3, for the quantitative variables, the means and s.d., or for the qualitative variables, the frequency distributions, are presented for the occlusal and functional variables, respectively.

Similar to the aforementioned pilot study, with a mean slide of $0.77 \mathrm{~mm}$ (s.d., $0.69 \mathrm{~mm}$ ) in the sleep bruxism group and a mean slide of $0.4 \mathrm{~mm}$ (s.d., $0.57 \mathrm{~mm}$ ) in the control group, the evaluation of the mean comparison between the two groups demonstrated a larger slide from $\mathrm{CO}$ to $\mathrm{MI}$ in sleep bruxism subjects $(P=0.008)$. Furthermore, considering the frequency distributions of the qualitative variables, the presence of a slide from CO to MI ( $P=0.008)$, as well as the presence of lesions related to lip and/or cheek $(P=0.013)$ biting was more frequently observed in sleep bruxism subjects than in controls. However, following Bonferroni adjustment none of the 16 occlusal and functional variables differed significantly between sleep bruxism subjects and non-sleep bruxism controls.

Table 1 Sociodemographic data of sleep bruxism subjects and controls

\begin{tabular}{lccc}
\hline Variable & Sleep bruxism group & Controls & $P$ \\
\hline Age & $29.09 \pm 4.65$ & $27.12 \pm 5.10$ & $0.065^{\mathrm{a}}$ \\
Gender & $39(67.2 \%) \mathrm{F} ; 19(32.8 \%) \mathrm{M}$ & $19(57.6 \%) \mathrm{F} ; 14(42.4 \%) \mathrm{M}$ & $0.357^{\mathrm{b}}$ \\
Education & $3 x_{1} ; 0 x_{2} ; 36 x_{3} ; 19 x_{4}$ & $2 x_{1} ; 2 x_{2} ; 24 x_{3} ; 5 x_{4}$ & $0.095^{\mathrm{b}, \mathrm{c}}$ \\
\hline
\end{tabular}

Following to Bonferroni adjustment, significance level amounted to $P<0.003$.

${ }^{a}$ Two-samples $t$-test; data are presented as mean \pm s.d.

${ }^{\mathrm{b}}$ Chi-square test.

${ }^{\mathrm{c}}$ Education was divided into four grades: $x_{1}, 10$ years school; $x_{2}, 12$ years school; $x_{3}$, 13 years school; $x_{4}, 18$ years school (university). 
Table 2 Functional and occlusal parameters of sleep bruxism subjects and controls (quantitative variables)

\begin{tabular}{lcrc}
\hline Variable/mm & Sleep bruxism group & \multicolumn{1}{c}{ Controls } & $P$ \\
\hline Overbite & $2.60 \pm 1.22$ & $3.02 \pm 1.38$ & $0.142^{\mathrm{a}}$ \\
Overjet & $2.73 \pm 1.26$ & $2.82 \pm 1.62$ & $0.732^{\mathrm{b}}$ \\
Maximum active mouth opening & $50.35 \pm 5.41$ & $51.83 \pm 6.47$ & $0.243^{\mathrm{a}}$ \\
Maximum active right movement & $9.32 \pm 2.39$ & $9.02 \pm 2.34$ & $0.558^{\mathrm{a}}$ \\
Maximum active left movement & $9.94 \pm 2.10$ & $9.79 \pm 2.48$ & $0.757^{\mathrm{a}}$ \\
Maximum active protrusive & $8.91 \pm 2.23$ & $8.21 \pm 1.94$ & $0.138^{\mathrm{a}}$ \\
$\quad$ movement & & & \\
Resiliency of the right TMJ & $0.58 \pm 0.29$ & $0.58 \pm 0.30$ & $0.882^{\mathrm{b}}$ \\
Resiliency of the left TMJ & $0.59 \pm 0.34$ & $0.56 \pm 0.28$ & $0.700^{\mathrm{b}}$ \\
CO to MI slide & $0.77 \pm 0.69$ & $0.40 \pm 0.57$ & $0.008^{\mathrm{b}}$ \\
\hline
\end{tabular}

Following to Bonferroni adjustment, significance level amounted to $P<0.003$.

${ }^{a}$ Two-samples $t$-test; data are presented as mean \pm s.d.

${ }^{\mathrm{b}}$ Mann-Whitney U-test; data are presented as mean \pm s.d.

Table 3 Frequencies of several functional and occlusal parameters. In this table the qualitative variables are presented.

\begin{tabular}{|c|c|c|c|}
\hline Variable & Sleep bruxism group & Controls & $P$ \\
\hline \multirow[t]{4}{*}{ Right canine $(n=68)$} & 17 Class I & 11 Class I & $0.455^{a}$ \\
\hline & 27 Class II & 9 Class II & \\
\hline & 3 Class III & 1 Class III & \\
\hline & 11 Missing & 12 Missing & \\
\hline \multirow[t]{4}{*}{ Left canine ( $n=68)$} & 24 Class 1 & 12 Class I & $0.707^{\mathrm{a}}$ \\
\hline & 22 Class II & 8 Class II & \\
\hline & 1 Class III & 1 Class III & \\
\hline & 11 Missing & 12 Missing & \\
\hline \multirow[t]{4}{*}{ Right first molar ( $n=65$ ) } & 14 Class I & 9 Class I & $0.324^{\mathrm{a}}$ \\
\hline & 19 Class II & 10 Class II & \\
\hline & 11 Class III & 2 Class III & \\
\hline & 14 Missing & 12 Missing & \\
\hline \multirow[t]{4}{*}{ Left first molar $(n=66)$} & 21 Class I & 11 Class I & $0.425^{a}$ \\
\hline & 14 Class II & 7 Class II & \\
\hline & 11 Class III & 2 Class III & \\
\hline & 12 Missing & 13 Missing & \\
\hline \multirow[t]{5}{*}{ Anterior crowding ( $n=68)$} & $0: n=9$ & $0: n=3$ & $0.644^{\mathrm{a}}$ \\
\hline & $1: n=34$ & $1: n=15$ & \\
\hline & $2: n=3$ & $2: n=3$ & \\
\hline & 3: $n=1$ & 3: $n=0$ & \\
\hline & 11 Missing & 12 Missing & \\
\hline $\begin{array}{l}\text { Presence of a CO to MI slide } \\
(n=91)\end{array}$ & $41(70.7 \%)$ & $14(42.4 \%)$ & $0.008^{a}$ \\
\hline Lip/cheek biting ( $n=91)$ & $16(27.6 \%)$ & $2(6.1 \%)$ & $0.013^{\mathrm{a}}$ \\
\hline
\end{tabular}

Following to Bonferroni adjustment, significance level amounted to $P<0.003$.

${ }^{\text {a }}$ Chi-square test.

\section{DISCUSSION}

The purpose of the present study was to verify the findings of a previous retrospective pilot study ${ }^{27}$ by means of a prospective controlled investigation including a larger sample size. The main result of the present investigation was that considering the 16 occlusal and functional parameters evaluated, sleep bruxism subjects and controls do not differ significantly. Indeed, similar to the forecited retrospective investigation, the same 16 occlusal and functional parameters have been used in the present study. Moreover, the values which have been recorded for these 16 variables in both studies move into a similar direction, however, following the Bonferroni adjustment each of the obtained differences between the sleep bruxism group and the controls failed to reach the level of statistical significance. For this reason, our hypothesis that there are no differences among a sample of sleep bruxism subjects and a non-sleep bruxism control group with respect to the occlusal and functional parameters evaluated could not be rejected.

When interpreting the underlying reasons for the observed discrepancies between the retrospective pilot study and the present prospective controlled investigation, methodological and statistical considerations need to be included, such as the prospective analysis of a larger sample size or the application of classical Bonferroni correction. ${ }^{37-38}$ The Kolmogorov-Smirnov test revealed that the variable length of a slide from $\mathrm{CO}$ to MI was not normally distributed. Consequently, the calculated s.d. were expected to be high. Generally, it is a fact that if a likewise large sample is investigated the scattering will be reduced and, thus, this will strengthen the reliability of the test used in the present study. From a critical point of view, the probability is, therefore, comparatively high that the effect which has been recorded in the preceding pilot study was by chance.

Comparisons of the present data with other previous investigations could not easily be performed due to diverse reasons. For instance, different criteria or methods have been applied for sleep bruxism diagnosis and, accordingly, this resulted in different sample compositions. ${ }^{13,39}$ Moreover, diverse studies vary regarding the parameters that have been recorded. For example, one investigation included a group of treated bruxism subjects, untreated bruxism subjects, and subjects with TMDs as well, whereas the details for sleep bruxism diagnosis have not been reported in detail. The evaluation of this more inhomogeneous sample revealed that $100 \%$ of the participants had laterotrusive interferences, $78 \%$ had mediotrusive interferences and 95.4\% showed premature contacts. ${ }^{39}$ Considering the before mentioned arguments, a comparison of this outcome with the present data could hardly be made. In another study, a clear description of the sample composition has been performed by using the polysomnographic criteria for sleep bruxism diagnosis. ${ }^{13}$ In this investigation, 26 occlusal and cephalometric measures have been evaluated in a sample of 10 sleep bruxism subjects and 10 controls. As result, none of the measures tested revealed a statistically significant difference between the two groups. On the basis of their results, authors concluded that the orofacial morphology of sleep bruxism subjects does not differ from that of non-sleep bruxism subjects. However, as the mentioned study had a retrospective design and, moreover, still included a sample size of 20 subjects, the authors point at its low statistical power. Taken these limitations into account, the authors emphasized the need to carry out further investigations in this field in terms of a larger sample and an experimental and prospective character. $^{39}$

In this context, attention should be drawn to the process of sleep bruxism diagnosis. Undoubtedly, due to the good performance of the validity parameters, to date, the laboratory polysomnographic recordings represent the highest standard for sleep bruxism diagnosis, ${ }^{29}$ but they are concomitantly associated with disadvantages which include technical complexity, limited availability, ${ }^{5-6}$ and the fact that they are time-consuming and cost-intensive. ${ }^{40}$ Similar to the abovementioned study, ${ }^{13}$ this is seen in comparatively small sample sizes of polysomnographic studies, ${ }^{41-42}$ which limits the applicability of this method, in particular, for clinical studies with larger sample sizes. ${ }^{6}$ As addressed above, when comparing the outcome of the aforementioned retrospective study with the present data, it must be indicated that both investigations have evaluated partially different variables and have used different assessment methods for sleep bruxism diagnosis. Notwithstanding these differences, both studies correspond with respect to the fact that none of the measures that have been recorded 
in the respective study revealed a statistically significant difference between sleep bruxism subjects and controls. For this reason, the results obtained in the present investigation were found as a reference that the occlusal and functional parameters evaluated do not differ between sleep bruxism subjects and non-sleep bruxism subjects.

Furthermore, as a possible consequence of myalgia due to sleep bruxism, a limitation of mouth opening and mandibular excursive movements in sleep bruxism subjects has also been reported. ${ }^{15,43}$ Since none of the variables regarding the mandibular movement showed a significant difference between the sleep bruxism group and the non-sleep bruxism controls, on the basis of the data derived from the present investigation, these movement limitations could not be confirmed as well. Moreover, if the present data are interpreted including physiologic mean values of mandibular movement capacity, ${ }^{44}$ the values that have been recorded in both the sleep bruxism group, as well as in the control group were located within normal range.

When interpreting the data of the present investigation, both the current knowledge on the development of sleep bruxism, as well as its possible effects ought to be taken into consideration. The main result of the present prospective study was that regarding 16 functional and/ or occlusal parameters sleep bruxism subjects did not differ significantly. Accordingly, this outcome supports the conclusion of previous authors that there is no proof for a role of dental occlusion and factors related to the anatomy of the orofacial skeleton in the aetiology of sleep bruxism. ${ }^{8}$ Moreover, as derived from the present results, the mandibular function does not appear to be involved too. Therefore, the authors of the present study may propose that the stomatognathic system, in particular, the dental occlusion should rather be viewed as a sort of effector organ of sleep bruxism activity which is most likely induced by a central causation. Usually, sleep bruxism is not aggravated by pain symptoms, ${ }^{16,45}$ however, as derived from the available research, a possible association between bruxism and certain subgroups of TMDs has been supposed. ${ }^{16-18,45-46}$ For this reason, it has to be asked at what point a stomatognathic system decompensates and turns to a disorder which requires a therapy. Further, some recent studies among the relationship of sleep bruxism and posture ${ }^{19-25}$ give an initial hint that consequences of sleep bruxism may be also detectable in other peripheral areas of the whole body. However, it should be pointed out that the last attempt requires further scientific evaluation to support a cause-effect relation due to diverse reasons (e.g., partly reported by means of case reports and studies including only small sample sizes). ${ }^{20,24}$ In the same way as regarding the stomatognathic system, one has to wonder among what circumstances further peripheral systems of the whole body decompensate and turn to a manifest disorder or reveal negative effects following to long-term sleep bruxism. Considering these questions, it appears advisable to turn one's attention closer to the individual adaptive capacity which has an impact on both the stomatognathic system and other peripheral systems of the human body. For an improved summary, these considerations were merged to a hypothetical model as illustrated in Figure 1. Likewise to other medical disciplines, ${ }^{4-50}$ this might offer the opportunity to a more sophisticated understanding why one subject with sleep bruxism will develop in addition to the immediate effects of sleep bruxism on the dental hard tissues a manifest TMD or, perhaps at a later date, further effects in other peripheral regions of the whole body and why another subject with sleep bruxism will not. Possible components which might influence the adaptive capacity are physiologic basics (such

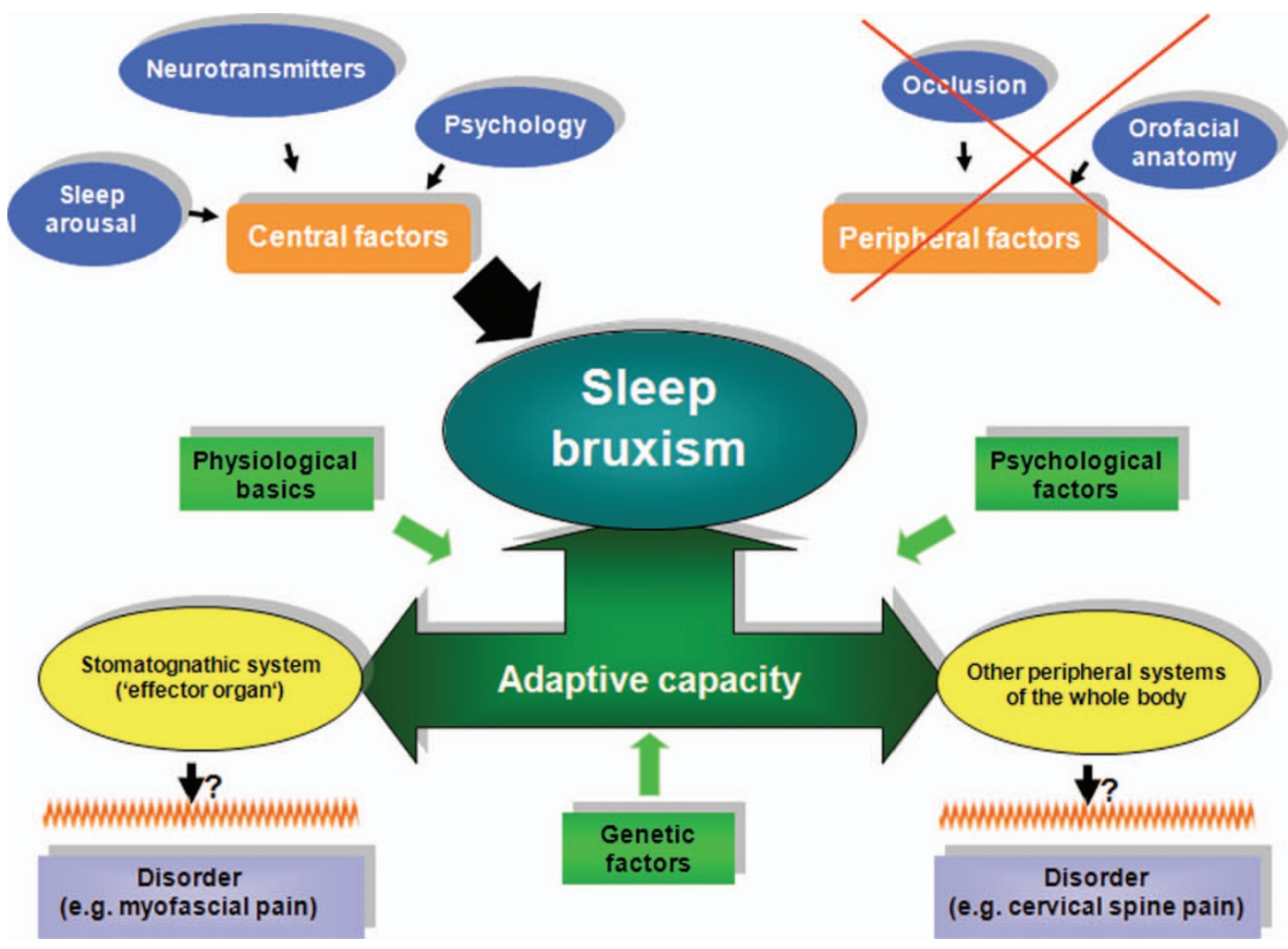

Figure 1 Schematic illustration which includes the current knowledge on the development of sleep bruxism and its possible effects on the stomatognathic system and/or other peripheral systems of the whole body. Moreover, to contribute to a more sophisticated understanding, the possible role of the adaptive capacity is embraced. 
as changes in motor unit recruitment patterns), psychological factors (such as vulnerability, resiliency), and genetic factors (such as predisposition).

\section{CONCLUSION}

With respect to the 16 occlusal and functional parameters evaluated, sleep bruxism subjects and controls did not differ significantly. Similar to the previous pilot study, a $0.4 \mathrm{~mm}$ larger slide from $\mathrm{CO}$ to $\mathrm{MI}$ has been observed in the sleep bruxism group, but following to Bonferroni adjustment this difference failed to reach the level of statistical significance. Keeping in mind the available literature indicating a possible association between sleep bruxism and certain subgroups of TMDs and a reference to possible effects of sleep bruxism on the posture, it has to be asked at what point a stomatognathic system or any other peripheral system of the whole body decompensates and turns to a disorder which requires a therapy. Embracing the role of the adaptive capacity of the stomatognathic system as one part of the human body into future investigations, will contribute to a more sophisticated understanding of sleep bruxism and its most likely multifactorial nature.

\section{ACKNOWLEDGEMENTS}

This study was supported in part by grants from the German Research Foundation (839/6-1, 839/6-2).

1 American Academy of Sleep Medicine. The international classification of sleep disorders, revised: diagnostic and coding manual. 2nd ed. Westchester: American Academy of Sleep Medicine, 2005.

2 Lavigne GJ, Kato T, Kolta A et al. Neurobiological mechanisms involved in sleep bruxism. Crit Rev Oral Biol Med 2003; 14(1): 30-46.

3 Lobbezoo F, van der Zaag J, van Selms MK et al. Principles for the management of bruxism. J Oral Rehabil 2008; 35(7): 509-523.

4 Mascaro MB, Prosdocimi FC, Bittencourt JC et al. Forebrain projections to brainstem nuclei involved in the control of mandibular movements in rats. Eur J Oral Sci 2009; 117(6): 676-684.

5 Seraidarian P, Seraidarian PI, das Neves Cavalcanti B et al. Urinary levels of catecholamines among individuals with and without sleep bruxism. Sleep Breath 2009; 13(1): 85-88.

6 Winocur E, Uziel N, Lisha T et al. Self-reported bruxism - associations with perceived stress, motivation for control, dental anxiety and gagging. J Oral Rehabil 2011; 38(1): 3-11.

7 Bader G, Lavigne G. Sleep bruxism; an overview of an oromandibular sleep movement disorder. Sleep Med Rev 2000; 4(1): 27-43.

8 Lobbezoo F, Naeije M. Bruxism is mainly regulated centrally, not peripherally. J Ora Rehabil 2001; 28(12): 1085-1091.

9 Manfredini D, Lobbezoo F. Role of psychosocial factors in the etiology of bruxism. $J$ Orofac Pain 2009; 23(2): 153-166.

10 Miller VJ, Yoeli Z, Barnea E et al. The effect of parafunction on condylar asymmetry in patients with temporomandibular disorders. J Oral Rehabil 1998; 25(9): 721-724.

11 Ramfjord SP. Bruxism, a clinical and electromyographic study. J Am Dent Assoc $1961 ; 62: 21-44$.

12 Young DV, Rinchuse DJ, Pierce CJ et al. The craniofacial morphology of bruxers versus nonbruxers. Angle Orthod 1999; 69(1): 14-18.

13 Lobbezoo F, Rompre PH, Soucy JP et al. Lack of associations between occlusal and cephalometric measures, side imbalance in striatal D2 receptor binding, and sleeprelated oromotor activities. J Orofac Pain 2001; 15(1): 64-71.

14 Lavigne GJ, Khoury S, Abe S et al. Bruxism physiology and pathology: an overview for clinicians. J Oral Rehabil 2008; 35(7): 476-494.

15 Attanasio R. An overview of bruxism and its management. Dent Clin North Am 1997 , 41(2): 229-241.

16 de Leeuw R. Orofacial pain: guidelines for assessment, diagnosis and management 4th ed. Chicago: American Academy of Orofacian Pain. Quintessence Publishing Co, Inc, 2008.

17 Lavigne GJ, Manzini C, Kato T. Sleep bruxism. In: Kryger MH, Roth T, Dement WC eds Principles and practice of sleep medicine. 4th ed. Philadelphia: Elsevier Saunders, 2005: 946-959.

18 Michelotti A, Cioffi I, Festa $\mathrm{P}$ et al. Oral parafunctions as risk factors for diagnostic TMD subgroups. J Oral Rehabil 2010; 37(3): 157-162.

19 Bracco P, Deregibus A, Piscetta R. Effects of different jaw relations on postural stability in human subjects. Neurosci Lett 2004; 356(3): 228-230.
20 Knutson GA. Vectored upper cervical manipulation for chronic sleep bruxism, headache, and cervical spine pain in a child. J Manipulative Physiol Ther 2003; 26(6): E16.

21 Kritsineli M, Shim YS. Malocclusion, body posture, and temporomandibular disorder in children with primary and mixed dentition. J Clin Pediatr Dent 1992; 16(2): 86-93.

22 Motta LJ, Martins MD, Fernandes KP et al. Craniocervical posture and bruxism in children. Physiother Res Int 2011; 16(1): 57-61.

23 Rodriguez K, Miralles R, Gutierrez MF et al. Influence of jaw clenching and tooth grinding on bilateral sternocleidomastoid EMG activity. Cranio 2011; 29(1): 14-22.

24 Strini PJ, Machado NA, Gorreri MC et al. Postural evaluation of patients with temporomandibular disorders under use of occlusal splints. J App/ Oral Sci 2009; 17(5): 539-543.

25 Velez AL, Restrepo CC, Pelaez-Vargas A et al. Head posture and dental wear evaluation of bruxist children with primary teeth. J Oral Rehabil 2007; 34(9): 663-670.

26 Michelotti A, Buonocore G, Manzo P et al. Dental occlusion and posture: an overview. Prog Orthod 2011; 12(1): 53-58.

27 Ommerborn MA, Giraki M, Schneider C et al. Clinical significance of sleep bruxism on several occlusal and functional parameters. Cranio 2010; 28(4): 238-248.

28 Ommerborn MA, Giraki M, Schneider C et al. A new analyzing method for quantification of abrasion on the Bruxcore device for sleep bruxism diagnosis. $J$ Orofac Pain 2005; 19(3): 232-238.

29 Lavigne GJ, Rompre PH, Montplaisir JY. Sleep bruxism: validity of clinical research diagnostic criteria in a controlled polysomnographic study. J Dent Res 1996; 75(1): 546-552.

30 Ommerborn MA, Schneider C, Giraki M et al. Effects of an occlusal splint compared with cognitive-behavioral treatment on sleep bruxism activity. Eur J Oral Sci 2007; 115(1): 7-14

31 Johansson A, Haraldson T, Omar R et al. A system for assessing the severity and progression of occlusal tooth wear. J Oral Rehabil 1993; 20(2): 125-131.

32 Anon. The glossary of prosthodontic terms. J Prosthet Dent 2005; 94(1): 10-92.

33 Kampe T, Hannerz H, Strom P. Five-year longitudinal recordings of functional variables of the masticatory system in adolescents with intact and restored dentitions. A comparative anamnestic and clinical study. Acta Odontol Scand 1991; 49(4): 239-246.

34 Kerr DA, Ash MMJ, Millard HD. Oral diagnosis. 6th ed. St Louis: Mosby, 1983: 180227.

35 Gerber A, Steinhardt G. Disturbed biomechanics of the temporomandibular joint. In: Gerber A, Steinhardt G eds. Dental occlusion and the temporomandibular joint. Chicago: Quintessence Publishing Co, Inc, 1990: 27-47.

36 Schmuth G. Befunderhebung und Systematik in der Kieferorthopädie. In: Schmuth G ed. Kieferorthopädie-Praxis der Zahnheilkunde 11. 3rd ed. München: Urban \& Schwarzenberg, 1994: 1-48.

37 Widmalm SE, Christiansen RL, Gunn SM. Oral parafunctions as temporomandibular disorder risk factors in children. Cranio 1995; 13(4): 242-246.

38 Zöfel P. Statistik verstehen. Munich: Addison-Wesley, 2002.

39 Yustin D, Neff $P$, Rieger MR et al. Characterization of 86 bruxing patients with longterm study of their management with occlusal devices and other forms of therapy. $J$ Orofac Pain 1993; 7(1): 54-60.

40 John MT, Frank H, Lobbezoo $\mathrm{F}$ et al. No association between incisal tooth wear and temporomandibular disorders. J Prosthet Dent 2002; 87(2): 197-203.

41 Kato T, Montplaisir JY, Guitard F et al. Evidence that experimentally induced sleep bruxism is a consequence of transient arousal. J Dent Res 2003; 82(4): 284-288.

42 Lobbezoo F, Lavigne GJ, Tanguay R et al. The effect of catecholamine precursor Ldopa on sleep bruxism: a controlled clinical trial. Mov Disord 1997; 12(1): 73-78.

43 Dao TT, Lund JP, Lavigne GJ. Comparison of pain and quality of life in bruxers and patients with myofascial pain of the masticatory muscles. J Orofac Pain 1994; 8(4): 350-356.

44 Hugger A, Schindler HJ. Unterkieferbewegungen und deren Simulation. In: Hugger A, Türp JC, Kerschbaum T eds. Curriculum Orale Physiologie. Berlin: Quintessenz Verlags-GmbH, 2006: 53-83.

45 Davis CE, Carlson CR, Studts JL et al. Use of a structural equation model for prediction of pain symptoms in patients with orofacial pain and temporomandibular disorders. $J$ Orofac Pain 2010; 24(1): 89-100.

46 Manfredini D, Lobbezoo F. Relationship between bruxism and temporomandibular disorders: a systematic review of literature from 1998 to 2008. Oral Surg Oral Med Oral Pathol Oral Radiol Endod 2010; 109(6): e26-e50.

47 Gaebel W, Zielasek J. Ätiopathogenetische Konzepte und Krankheitsmodelle in der Psychiatrie. In: Möller HJ, Laux G, Kapfhammer HP eds. Psychiatrie und Psychotherapie. 3rd ed. Heidelberg: Springer, 2008: 29-54.

48 Anderson V, Spencer-Smith M, Wood A. Do children really recover better? Neurobehavioural plasticity after early brain insult. Brain 2011; 134(Pt 8): 2197 2221.

49 Eisenberg L. Psychiatry and society: a sociobiologic synthesis. N Eng/ J Med 1977; 296(16): 903-910.

50 Morton GJ, Cummings DE, Baskin DG et al. Central nervous system control of food intake and body weight. Nature 2006; 443(7109): 289-295. Attribution-NonCommercial-NoDerivative Works 3.0 Unported License. To view a copy of this license, visit http:// creativecommons.org/licenses/by-nc-nd/3.0 